\title{
REFERENTES PARA LA ORIENTACIÓN DEL APRENDIZAJE DESDE LA PERSPECTIVA DEL APRENDIZAJE AUTORREGULADO
}

\author{
REFERENTS FOR LEARNING REGULATING FROM THE \\ SELF-REGULATED LEARNING PERSPECTIVE \\ José Manuel Suárez Riveiro*, Daniel Anaya Nieto** \\ Universidad Nacional de Educación a Distancia \\ y Ana Patricia Fernández Suárez***
}

\section{RESUMEN}

Durante los últimos años ha surgido el concepto de Aprendizaje Autorregulado (AAR) generando una línea de investigación que pretende aunar los conocimientos y experiencia existentes en relación con las variables cognitivas, afectivo-motivacionales y del entorno de aprendizaje. Dicho constructo, pese a estar todavía en proceso de definición y explicación, puede proporcionar una buena base teórica para la comprensión y evaluación del proceso de aprendizaje de los estudiantes. En este sentido, pretendemos destacar los grandes rasgos que van a conducir en el futuro a la elaboración de recursos dirigidos a su evaluación. Así, en este trabajo presentamos sus principales características, componentes, líneas actuales de investigación e instrumentos que se sitúan próximos a su evaluación. Con todo ello, se pretende ayudar al desarrollo de los estudiantes, desde los centros educativos, en lo que tradicionalmente se ha venido denominando como "aprender a aprender".

Palabras clave: Aprendizaje autorregulado, evaluación, estrategias, motivación.

* José Manuel Suárez Riveiro es Doctor en Psicopedagogía y profesor en la Facultad de Educación de la U.N.E.D., desarrollando su actividad docente e investigadora en el Departamento de Métodos de Investigación y Diagnóstico en Educación II (Orientación Educativa, Diagnóstico e Intervención Psicopedagógica). Sus líneas de investigación se centran en torno al aprendizaje autorregulado, las estrategias y la motivación en los procesos de aprendizaje y estudio.

** Daniel Anaya Nieto es profesor en la Facultad de Educación de la U.N.E.D. Sus líneas de investigación, actualmente, están centradas en la elaboración y validación de recursos para el diagnóstico y la intervención educativa.

*** Ana Patricia Fernández Suárez es maestra y licenciada en Psicopedagogía. Su actividad investigadora se centra en torno a las estrategias autorreguladoras del aprendizaje. 


\begin{abstract}
The concept of self-regulated learning has emerged during the last years generating an investigation line which tries to join the knowlege and investigation on cognitive, affective-motivational and learning environment variables. This construct, althought being under a process of definition and explanation, can provide a good theoretical basis for the understanding and evaluation of students' learning process. In this way, we try to underline the main features which in the near future will lead us to the elaboration of resources for its evaluation. Thus, in this work we present its main characteristics, components, current investigation lines and instruments near its evaluation. All in all, we try to help students development, in educational centres, of what traditionally has been called "learning to learn".
\end{abstract}

Key words: Self-regulated learning, evaluation, strategies, motivation.

\title{
Aproximación al concepto de aprendizaje autorregulado
}

Muchas han sido las teorías que tradicionalmente han intentado explicar el proceso de aprendizaje en los estudiantes. Probablemente, uno de los cambios más relevantes para dicha explicación ha sido el producido por la consideración de los procesos de pensamiento del alumno. En algunas de las teorías más tradicionales el profesor era el agente que prescribía y dirigía lo que los estudiantes debían hacer para alcanzar los objetivos, los cuales también eran presentados por el profesor. Este tipo de enseñanza conducía la mayoría de las veces a un conocimiento aislado e inerte y por tanto carente de funcionalidad. Así, frente a la tradicional manera de entender el proceso de enseñanza/aprendizaje como un proceso unidireccional, de consumo de conocimientos, pasivo, del profesor al alumno, se tiende a considerar una serie de elementos significativos que se encuentran en la mente del alumno (p.e., sus atribuciones, conocimientos previos, autoconcepto, metas académicas, estrategias de aprendizaje, etc.) y que comprenden tanto elementos cognitivos como elementos afectivos-motivacionales, los cuales actúan de forma conjunta y entrelazada. Se pasa así a considerar al alumno como agente activo y autodirigido (Beltrán, 1993), construyendo, modificando y utilizando su conocimiento para interpretar situaciones en un determinado dominio y actuar dentro de él (Vermunt y Verloop, 1999).

En este sentido, a lo largo de las dos últimas décadas han surgido distintos términos para referirse al aprendizaje que es activado por el propio individuo. Entre ellos podemos destacar las denominaciones de aprendizaje autónomo, aprendizaje autodirigido, aprendizaje independiente o autoaprendizaje. Pero ha sido el término aprendizaje autorregulado (self-regulated learning) (Corno, 1986; Zimmerman y Schunk, 1989) el que ha alcanzado una mayor difusión tanto desde la perspectiva teórica como en las distintas investigaciones realizadas, constituyéndose en la actualidad en un amplio campo de estudio que está en continua expansión. Desde estas posiciones se intenta explicar cómo y por qué el estudiante rinde (o no), incluso en aquellas situaciones en las que su nivel intelectual, ambiente sociocultural y calidad de la enseñanza predicen lo contrario. Zimmerman (1994) describe cómo algunos alumnos pertenecientes a minorías en situaciones de desigualdad llegan a triunfar gracias a su persistencia, su compromiso, su fuerte sentido de eficacia, su uso del tiempo, de métodos de aprendizaje y su dirección de metas. Comenta también este autor que existen evidencias con relación a que la principal causa de fracaso es la incapacidad de los estudiantes para autocontrolarse a sí mismos de forma efectiva. Siendo estos estudiantes que fracasan más impulsivos, más ansiosos, con una menor autoestima, influenciados en mayor 
medida por factores extrínsecos, con metas académicas más bajas, menos cuidadosos con la evaluación de sus habilidades; pero, además, son más autocríticos y menos autoeficaces sobre su ejecución, tendiendo a desistir más fácilmente.

Según Winne (1995), los aprendices autorreguladores de su aprendizaje cuando se ocupan de una tarea que implica ir paso a paso, reparten el tiempo en diversos tópicos:

a) buscar y recuperar información en el campo de la tarea,

b) supervisar su implicación en relación con las metas e identificar las desviaciones desde las rutas que planificaron, para así continuar en el esfuerzo por alcanzar dichas metas,

c) hacer ajustes específicos o bien rediseñar los planes estratégicos de estudio basándose en los juicios sobre qué caminos tienen una mayor posibilidad de éxito para las múltiples submetas específicas, así como para las metas generales más distantes, y

d) revisar el conocimiento de campo y las creencias sobre la autocompetencia.

En el caso de surgir determinados obstáculos, el estudiante autorregulado puede hacer una o varias modificaciones:

a) las metas iniciales pueden ser reajustadas o incluso abandonadas,

b) las razones que motivan la persistencia en el estudio pueden ser redirigidas/reevaluadas, y

c) la afectividad que acompaña a todo el proceso puede ser que necesite ser disciplinada.

Más aún, si la supervisión de los efectos de estos ajustes revela que el progreso es lento, se interrumpe o se reincide, pueden volver a ellos o intentar adaptar las estrategias que están empleando. Pueden intentar inventar nuevos significados para superar los obstáculos o, en casos razonables, incluso llegar a abandonar la tarea por completo.

Es pues, el AAR una aptitud deliberativa, de juicio, y adaptativa en proporciones expertas (Winne, 1995) en la que los criterios claves para considerar una conducta relacionada al desarrollo de un AAR es que las metas que la conducen sean metas personales y que para ello el estudiante tenga en cuenta las condiciones de su entorno (Boekaerts, 2001).

\section{Aspectos a considerar en la evaluación del aprendizaje autorregulado}

En relación con las descripciones que acabamos de comentar sobre el proceso seguido durante la autorregulación por parte del estudiante, cabe decir que habitualmente se hace referencia a una conceptulización restringida sobre los aspectos que comprende el AAR. Así, se alude a la gestión de los esfuerzos y a la planificación, supervisión y regulación de las estrategias cognitivas más simples que el alumno utiliza en su aprendizaje. Pero el AAR también comprende otros aspectos de gran relevancia para el aprendizaje como es el caso de la motivación. En este sentido, la forma más habitual de considerar el componente motivacional en los diversos estudios desarrollados se ha vinculado a su establecimiento como determinante del componente estratégico, tanto en el ámbito cognitivo como metacognitivo o conductual, proporcionando las variables motivacionales el deseo, esfuerzo y compromi- 
so necesario para llevar a cabo las estrategias seleccionadas. Sin embargo, casi se ha obviado la regulación por parte del estudiante de su motivación, es decir, la regulación de su propio compromiso y deseo de esforzarse y persistir ante una determinada tarea académica (Wolters, 1998).

Para Pintrich (1995), el AAR se caracteriza por incluir la activación y dirección de metas y el autocontrol de la conducta, de la motivación y de la cognición para las tareas académicas por parte de un estudiante. Tres son los componentes principales del AAR que se pueden deducir de dicho planteamiento. El primero es que los aprendices autorreguladores intentan controlar su conducta, su motivación, su afecto y su cognición. Para su explicación, el autor recurre al ejemplo de un termostato, el cual regula la temperatura de la habitación mediante la supervisión de la temperatura actual y su ajuste a la temperatura seleccionada previamente. De la misma forma que el termostato, los estudiantes pueden supervisar tres aspectos generales, que son su propia conducta, su motivación y su cognición, y así regularlas y ajustarlas según las demandas de la situación. La autorregulación de la conducta incluye el control activo de varios recursos que el estudiante tiene disponibles, como son su tiempo de dedicación, su entorno de trabajo y la búsqueda de ayuda en compañeros y profesores. La autorregulación de la motivación y del afecto incluye el control y cambio de creencias motivacionales, tales como la autoeficacia, las atribuciones, el interés o la orientación de meta, que le permiten adaptarse a las demandas planteadas. Así, los estudiantes pueden aprender cómo controlar sus emociones y afectos, tales como la ansiedad, de cara a mejorar su aprendizaje. Y por último, la autorregulación de la cognición que incluye el control de varias estrategias cognitivas, como son el uso de estrategias de procesamiento superficial o profundo que favorecen el aprendizaje y la ejecución del estudiante.

La segunda característica descrita por Pintrich es la existencia de alguna meta que el estudiante persigue. Gracias a esta meta el estudiante posee una norma que le permite supervisar y juzgar su propia ejecución y así poder hacer los ajustes necesarios. De esta forma, los aprendices autorreguladores de su aprendizaje poseen metas académicas adaptativas, persistiendo en sus esfuerzos con el fin de alcanzarlas (Pintrich y García, 1991; Schunk, 1994).

La tercera característica del AAR es que es en el propio estudiante y no en otra persona (profesores, padres...) donde debe surgir el control de sus acciones. Es decir, su conducta no debe darse como respuesta a los requerimientos de otros. Es de esta tercera característica de donde surge el prefijo "auto" del término AAR.

En relación con esto último, es necesario hacer una distinción que puede llevar a error en la caracterización de la autorregulación. Nos referimos a la distinción de una misma conducta como respuesta autorreguladora o como, simplemente, una forma más convencional de aprendizaje. Para ello, Zimmerman (1994), sugiere que el criterio de elección o control por parte del individuo es esencial para el ejercicio de la autorregulación. Así, sería necesario dar la posibilidad de aprendizaje o ejecución que prefieran, de cara a poder hacer una inferencia que vaya más allá de si los estudiantes pueden utilizar una estrategia particular, resultado de aprendizaje, a adicionalmente determinar si ven su valor personal y así iniciar y controlar su uso, como resultado de autorregulación. En esta misma línea, Boekaerts (2001) considera la necesidad de que las metas del estudiante sean personales. De este modo, una misma conducta podría ser caracterizada como autorreguladora o simplemente como auto- 
control, en función del tipo de meta que el estudiante persiga. Sin embargo, también es cierto que podríamos argumentar que una determinada "meta externa" puede ser apropiada por el estudiante. En esta dirección resulta interesante la clasificación que presentan Sheldon y Elliot (1998), al diferenciar metas personales autónomas y metas controladas. Dentro de las primeras distinguen dos formas: una intrínseca, la cual surge del interés personal y que resulta agradable, autónoma y autointegrada, y otra identificada, que pese a no resultar agradable se ajusta a los valores y creencias del individuo. Por su parte, las metas controladas tendrían también dos formas: una extrínseca, que surge debido a las contingencias contextuales con el fin de conseguir una recompensa, y otra interiorizada que tiene como fin que el individuo no se sienta culpable o ansioso por no realizar una determinada conducta. Así pues, según los resultados obtenidos por estos autores, serán las metas personales autónomas las que predigan el logro y no lo hagan las controladas. Ello es debido a que las metas autónomas son más fáciles de proteger y mantener, posibilitando los continuos esfuerzos necesarios para alcanzar el logro pretendido (y el AAR). Mientras que los individuos que mantengan metas controladas, pese a poder tener la intención de trabajar duro, no consiguen sus pretensiones por no posibilitar estas metas la continuidad necesaria de sus esfuerzos. Vemos pues, lo relevante de las metas académicas como variable motivacional para el desarrollo de un adecuado nivel de AAR (ver Suárez, González, Abalde y Valle, 2001; Suárez, González y Valle, 2001).

Con todo lo tratado hasta el momento, podemos establecer que el planteamiento del AAR proporciona una visión global de las variables, tanto cognitivas como afectivo-motivacionales, que inciden en el desarrollo de lo que debe constituirse en un adecuado proceso de aprendizaje. Por tanto, dicho planteamiento servirá a los profesionales de recurso a la hora de desarrollar el proceso diagnóstico y orientador sobre el aprendizaje del estudiante, en el que las variables anteriormente citadas sean los principales referentes, y que han de estar en todo momento complementadas por otras variables de tipo contextual. En este sentido, habitualmente el AAR ha sido referido a un proceso caracterizado por la presencia de dos caras de una misma moneda. Es decir, la autorregulación cognitiva por un lado y la autorregulación motivacional por otro, obviándose así el entorno en el cual se produce el proceso de aprendizaje, el cual determina en la mayoría de los casos cómo se producirá dicho proceso. Así, el AAR surge del continuo intercambio entre los estudiantes y los elementos educativos de amplios entornos, los cuales incluyen a los adultos (principalmente profesores y padres) y a los iguales. Del mismo modo, habrá que considerar los diferentes curricula llevados a cabo y las actividades que proporcionan (Corno, 1995). Se considera pues, que los estudiantes autorreguladores también se pueden caracterizar por su capacidad para controlar y gestionar su entorno académico, social y físico en relación con su propio proceso de aprendizaje.

Con objeto de facilitar la comprensión, tanto de las variables que intervienen como de las relaciones que entre ellas se establecen, se han desarrollado diversos modelos en torno al aprendizaje, la motivación y las estrategias (p.e., Boekaerts, 1996; Borkowski y Muthukrishna, 1992; Garcia y Pintrich, 1994). A continuación presentamos nuestro propio planteamiento, el cual no constituye un sumario de hallazgos empíricos, sino un modelo sobre el cual trabajar (ver Fig. 1), cuya principal característica es la consideración de tres componentes relacionados a lo cognitivo, lo afectivo-motivacional y a los recursos y entorno del estudiante. 


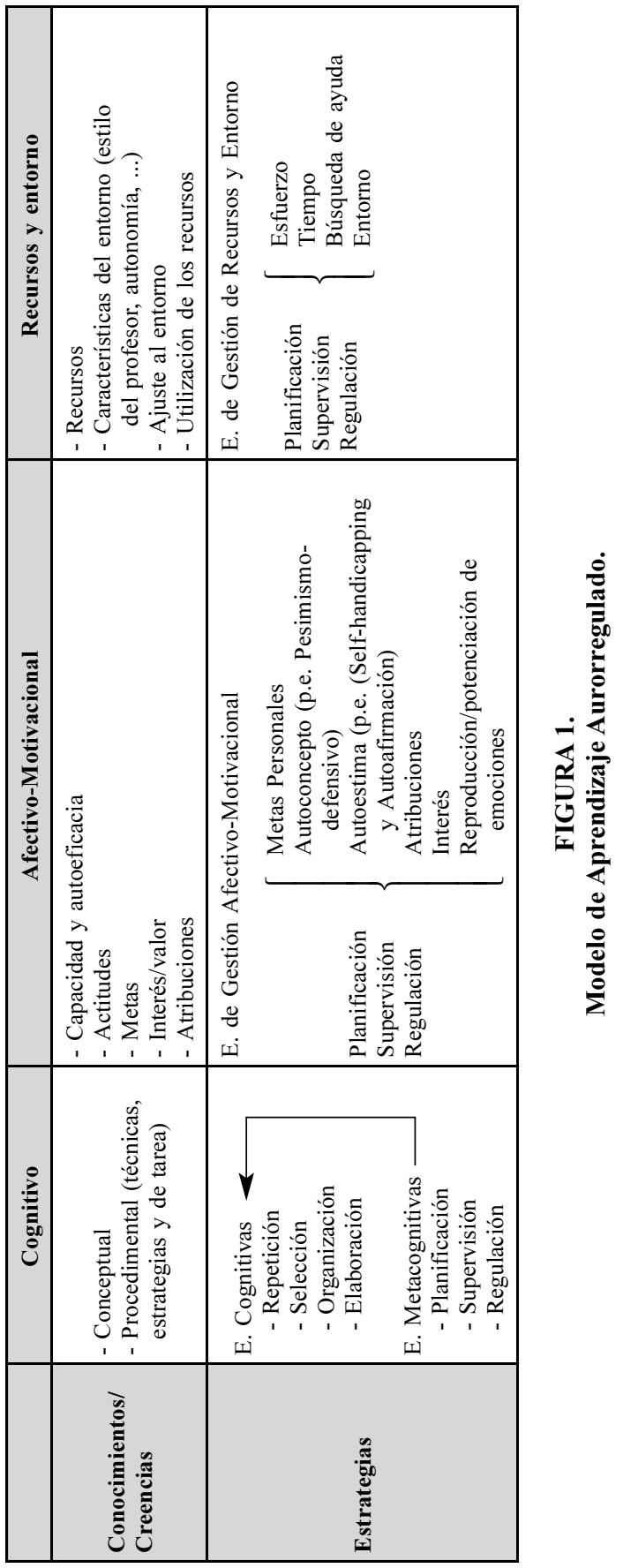


El modelo presentado establece dos niveles y tres dominios. El primer nivel hace referencia a los conocimientos o creencias presentes en el estudiante. Dentro de dichos conocimientos o creencias podemos diferenciar aquellos relacionados al ámbito cognitivo, al afectivo-motivacional y a las variables del entorno y los recursos del estudiante. Dentro del dominio cognitivo han de diferenciarse a su vez los conocimientos de tipo conceptual y los conocimientos procedimentales. Los primeros hacen referencia a la materia propia de estudio y los segundos se refieren a cómo proceder para alcanzar una determinada meta. A grandes rasgos, cabe comentar que los primeros no tienen por que ser acertados, dando lugar en muchos casos a conocimientos previos erróneos, y que los segundos, pese a ser conscientes pueden no llegar a ser aplicados.

Los conocimientos ligados al dominio afectivo/motivacional incluyen las creencias que el estudiante tiene sobre su capacidad, eficacia y metas. Es particularmente destacable la diferenciación entre la capacidad que el sujeto cree que puede tener para aprender de la que cree que puede tener para rendir. Así, puede darse el caso de un estudiante con alta capacidad para aprender pero que, sin embargo, alcanza un bajo rendimiento. Es probablemente el caso de aquellos estudiantes capaces pero poco motivados. Pero el objeto indirecto de conocimiento en este dominio no es únicamente el propio estudiante, también se incluyen los conocimientos y creencias que éste puede tener sobre su actitud ante aspectos tales como la materia de estudio o el tipo de actividad a desarrollar. Paralelamente al anterior dominio también podemos incluir aquí los conocimientos del estudiante sobre cómo proceder para incidir sobre determinados aspectos afectivos o motivacionales. Así, un estudiante puede tener una baja percepción sobre su competencia en una determinada materia, pero saber cómo puede favorecer que dicha percepción sea más positiva. Sin embargo, debemos ser conscientes de que dicho conocimiento no implica su aplicación, es decir la ejecución de la estrategia concreta y de las cuales nos ocupamos en el segundo nivel del modelo. Se incluyen también dentro de este dominio afectivo-motivacional variables tan relevantes como la orientación a metas, los intereses y el valor otorgado a la materia.

El tercer dominio al que se refieren los conocimientos y creencias de los estudiantes es el relativo a los recursos y entorno de aprendizaje. Aquí también se puede hacer una diferenciación pareja a la realizada en los dominios anteriores. Así, se puede hacer referencia al conocimiento del estudiante sobre las características del entorno, lo cual incluye el estilo de enseñanza del profesor, el grado de autonomía de que dispone, las posibilidades que le puede ofrecer su familia, las dotaciones existentes en su entorno, centro, hogar, ... Pero también debemos de diferenciar los conocimientos del alumno sobre cómo ajustar su aprendizaje al entorno. Así, el estudiante puede no disponer de un adecuado espacio de estudio, o de un profesor que no le permita desarrollar sus intereses, pero que en contrapartida sepa cómo conseguir que su familia o profesor favorezcan su estudio o intereses.

El segundo nivel del modelo propuesto hace referencia a los distintos tipos de estrategias de las que el estudiante puede disponer. Dentro de las estrategias referidas al ámbito cognitivo podemos diferenciar las estrategias propiamente cognitivas de las estrategias metacognitivas. Las estrategias cognitivas comprenden aquellas actividades que el estudiante utiliza para el procesamiento de la materia de estudio y que determinan de forma directa los resultados del aprendizaje, en referencia a los cambios en sus conocimientos sobre dicha materia. Se considera que el estudiante realiza un aprendizaje significativo cuando sitúa dicho conocimiento de una forma coherente en su sistema cognitivo. La forma en la que trate 
esa nueva información vendrá determinada por el tipo de estrategias que el alumno utilice, modo que las estrategias que el alumno utilice influyen sobre cómo procesa la información y el tipo de aprendizaje a que da lugar. Según Pintrich y De Groot (1990) las estrategias cognitivas constituyen uno de los componentes del AAR, que los estudiantes utilizan para aprender, recordar y comprender el material. Para Boekaerts (1996), el término "estrategia cognitiva" es utilizado para referirse a los procesos cognitivos y la conducta que los estudiantes utilizan durante las experiencias de aprendizaje para completar una asignación o realizar una meta implicada en la tarea académica.

Diferentes tipos de estrategias cognitivas han sido discutidos en la literatura. Siguiendo el trabajo de Weinstein y Mayer (1986), podemos considerar las estrategias de repetición, elaboración y organización que están relacionadas a la ejecución académica en el estudio. Estas estrategias pueden ser aplicadas a tareas simples de memoria (recordar palabras, listas, información, etc.) o a tareas más complejas que requieren comprensión de la información (comprensión de un texto) y no únicamente recordarla. Otros investigadores utilizaron categorizaciones diferentes, para agrupar diversas estrategias, que se solapan parcialmente (p.e., Entwistle, 1995; Schmeck y col., 1991; Vermunt y Verloop, 1999).

Tal como hemos visto son las estrategias cognitivas las que se ocupan de la ejecución del "aprendizaje", pero serán las estrategias autorreguladoras las que planifiquen, supervisen y regulen dicho aprendizaje. Así, a medida que los estudiantes crecen las estrategias cognitivas aumentan en número y se adecuan a las demandas de las tareas, lo cual lleva a que se desarrollen habilidades procedimentalizadas, así como a que puedan adaptar sus estrategias cognitivas en situaciones en las que se producen desajustes entre las estrategias cognitivas activadas y las demandas situacionales (Boekaerts, 1996). Además de las estrategias cognitivas, el conocimiento metacognitivo de los estudiantes y el uso de estrategias metacognitivas pueden tener una importante influencia sobre su logro (p.e. Zimmerman y Martinez-Pons, 1988; Boekaerts, 1996; Bouffard, Boisvert, Vezeau y Larouche, 1995). Para Boekaerts (1996), las estrategias autorreguladoras, o como ella las denomina "estrategias autorreguladoras cognitivas" se refieren a procesos cognitivos y la conducta que es engranada especialmente hacia la realización de metas marcadas y hacia la regulación de unas determinadas actividades de cara a alcanzar dichas metas.

La mayoría de los modelos de control metacognitivo incluyen cuatro tipos generales de estrategias: planificación, supervisión, regulación y evaluación que parecen estar altamente relacionadas y que ayudan a los estudiantes a guiar y dirigir su cognición (Boerkaerts, 1996; Corno, 1986; Garcia y Pintrich, 1994; Pintrich y DeGroot, 1990; Pintrich y García, 1991; Pintrich, Roeser y De Groot, 1994; Vermunt y Verloop, 1999; Zimmerman y Martínez-Pons, 1986).

El tercer tipo de estrategias lo constituyen las estrategias motivacionales. A este respecto cabe comentar, en primer lugar, que la investigación reciente indica que los estudiantes que están más interesados, que se sienten más a gusto, que están intrínsecamente motivados, con mayor probabilidad gastarán mayor cantidad de esfuerzo en las tareas que aquellos estudiantes que se sienten ansiosos, incómodos y están extrínsecamente motivados. Así pues, no es únicamente el componente cognitivo de lo que nos debemos ocupar, sino que también hemos de tener en cuenta el componente motivacional, pues éste permitirá el óptimo desempeño del componente cognitivo, elicitando cogniciones y emociones positivas. Sin embargo, no hemos de confundir la motivación con las estrategias motivacionales. Una 
clara diferenciación la plantea Zimmerman (1994) quien distingue el término motivación intrínseca del término automotivado. A través del primero, se asume el deseo del estudiante de continuar su estudio en ausencia de control externo directo. Prefiere este autor el término automotivado debido a que el término motivación intrínseca asume que la motivación es derivada de la tarea, mientras que con el término automotivado la motivación se deriva de sus percepciones de autoeficacia y uso de procesos autorreguladores durante el aprendizaje. Según Boekaerts (1996), hay que distinguir entre rasgos personales que representan inclinación de los estudiantes a comprometerse en el aprendizaje escolar y la sensibilidad selectiva o disponibilidad momentánea de los estudiantes para situaciones de aprendizaje específicas. La disposición momentánea y atención para lograr el dominio, completar una tarea o cumplir una meta de aprendizaje es el resultado de estrategias de motivación aplicadas en situaciones de aprendizaje.

$\mathrm{Al}$ igual que las estrategias cognitivas, las estrategias motivacionales son usadas por los estudiantes durante las experiencias de aprendizaje, siendo utilizadas en este caso para enfrentarse a las emociones que surgen durante su aprendizaje y pudiendo ser, al igual que las estrategias cognitivas, automáticas o bajo el control del estudiante. Pero, a diferencia de las primeras, no están comprometidas con el procesamiento del contenido, aunque sí favorecen estados favorables de compromiso con el aprendizaje, o bien ayudan a prevenir eventos no deseables y resultados desfavorables. Es decir, la utilización de este tipo de estrategias motivacionales por parte del estudiante conlleva consecuencias motivacionales, cognitivas y afectivas, que pueden resultar positivas, neutras o negativas para el adecuado progreso del proceso de aprendizaje.

Seguramente, son las estrategias motivacionales el componente estratégico menos estudiado en los distintos trabajos desarrollados en torno al AAR. Dentro de las estrategias motivacionales podemos encontrar acciones tales como la creación de una intención de aprendizaje, las atribuciones prospectivas y retrospectivas, la evitación del esfuerzo y la utilización de estrategias de enfrentamiento para alterar estresores y para reducir las emociones negativas. Otras estrategias motivacionales más específicas son las estrategias de ponerse trabas (self-handicapping), optimismo, pesimismo defensivo, autoafirmación y evitación de expectativas (sandbagging).

Un cuarto y último tipo de estrategias son las referidas a los recursos y el entorno del estudiante. Con ello nos referimos a las estrategias que los estudiantes utilizan para gestionar sus esfuerzos y su medio (Corno, 1986; Pokay y Blumenfeld, 1990; Zimmerman y Martinez-Pons, 1986). El esfuerzo es un aspecto ampliamente relacionado con la motivación pero que el estudiante puede planificar, supervisar y regular en función de sus metas. Dicho esfuerzo puede ser planteado tanto en el ámbito cualitativo como en el ámbito cuantitativo. El cualitativo se refiere a la calidad y profundidad de las tareas desarrolladas, mientras que el cuantitativo al tiempo empleado. Con respecto a la gestión del medio, éste incluye el entorno de estudio y a los otros, donde se incluyen profesores, padres y compañeros. En línea con un enfoque general adaptativo de aprendizaje, se puede asumir que estas estrategias de gestión de recursos ayudan a los estudiantes a adaptarse a sus entornos, así como a modificarlos para ajustarlos a sus metas y necesidades (ver Suárez y col., 2001). Las estrategias para autorregular estos componentes pueden ser modelados por el profesor mediante ejercicios específicos de entrenamiento. Por ejemplo, con alumnos que tienen problemas en el manejo del tiempo se les puede enseñar la planificación del tiempo, y proporcionar oportu- 
nidades para su control (primero bajo condiciones estructuradas y posteriormente en condiciones más dinámicas).

Con todo ello, se establece que tanto la evaluación diagnóstica como las investigaciones a desarrollar en torno al concepto de AAR, habrán de tener en cuenta el estudio de los diversos tipos de conocimientos, creencias y estrategias utilizadas por los estudiantes, considerando situaciones que impliquen tanto contenidos específicos como variables afectivomotivacionales, contextuales y nivel de desarrollo de capacidades y habilidades respecto a su edad.

\section{Recursos para la evaluación del aprendizaje autorregulado}

Pese a que el individuo ha de ser considerado como un todo, en el que las distintas variables están en compleja interacción, con fines diagnósticos cabe la posibilidad de acotar áreas con objeto de su estudio (Anaya, 2002). Así, a grandes rasgos, podemos diferenciar entre diversos tipos de variables que inciden en el aprendizaje. En primer lugar, es preciso diferenciar entre variables del entorno y variables personales. Es en relación con estas últimas donde se sitúa el constructo de AAR, a la vez que se relaciona ampliamente con las variables del entorno del estudiante.

La caracterización ofrecida previamente sobre el AAR hace referencia inmediata a los pensamientos del alumno. Es por ello que las técnicas más adecuadas para su evaluación son las de autoinforme, las cuales comprenden la entrevista; los cuestionarios, inventarios y escalas; los autorregistros y las técnicas de pensamiento en-voz-alta.

Hasta la actualidad todavía no se ha desarrollado un único instrumento lo suficientemente sólido, ya sea por razones de constancia en los datos recogidos sobre su fiabilidad y validez, o por la falta de consideración de algunas de las variables anteriormente comentadas en torno al constructo de AAR. Sin ánimo de exhaustividad, a continuación recogemos algunos de los instrumentos que pueden resultar útiles en la evaluación del AAR y de algunas de las variables más relevantes que comprende.

Dentro de los cuestionarios más relevantes en el campo que nos ocupa destaca el Cuestionario de Estrategias Motivadas para el Aprendizaje (MSLQ) de Pintrich, Smith, Garcia y Mckeachie (1991), el cual fue traducido y adaptado al castellano por Roces, Tourón y González (1995) dando lugar a dos versiones, los Cuestionarios de Estrategias de Aprendizaje y Motivación I y II (CEAM I y II). La primera versión está dirigida a la evaluación de la motivación y las estrategias de un alumno dentro de una asignatura concreta, mientras que la segunda está dirigida a su evaluación con respecto a un curso completo. Partiendo de un modelo teórico que integra tanto componentes cognitivos como afectivo-motivacionales, evalúa la orientación motivacional y uso estratégico de los estudiantes. Este instrumento está compuesto, por un lado, de tres escalas y seis subescalas motivacionales: La escala de valor, que incluye tres subescalas (orientación a metas intrínsecas, orientación a metas extrínsecas y valor de la tarea) y que se centra en las razones del estudiante para comprometerse en las tareas académicas; la escala de expectativas, formado por dos subescalas (creencias de control del aprendizaje y autoeficacia para el aprendizaje y el rendimiento), que se centra en las creencias del estudiante sobre si considera que puede realizar las tareas o no; y la escala de afectividad, que incluye una única subescala (ansiedad) sobre la preocu- 
pación e inquietud del estudiante ante los exámenes. Y, por otro lado, consta de nueve subescalas cognitivas, las cuales se agrupan en tres escalas: una escala de estrategias cognitivas, formado por cuatro subescalas (estrategias de repetición, elaboración, organización y pensamiento crítico) relativas al uso de estrategias simples y complejas del procesamiento de la información; una segunda escala de estrategias metacognitivas, formado por una única subescala (autorregulación metacognitiva), que se centra en las estrategias que utiliza el estudiante para controlar y regular su propia cognición; y una tercera, y última escala, de estrategias de control de los recursos, compuesto a su vez por cuatro subescalas (tiempo y lugar de estudio, regulación del esfuerzo, aprendizaje con otros compañeros y búsqueda de ayuda) sobre las estrategias reguladoras de los estudiantes para controlar otros recursos que no pertenecen a la cognición. Se matiza, además, que las 15 subescalas pueden ser utilizadas conjuntamente o de forma separada según las necesidades que establezca el investigador o educador.

Otros instrumentos a destacar entre los diversos disponibles para la evaluación de las estrategias y técnicas de estudio, y de la motivación son:

- El Inventario de Habilidades de Aprendizaje y Estudio (LASSI) (Weinstein, 1987) cuya finalidad es evaluar tanto estrategias y habilidades de aprendizaje como aspectos afectivo-motivacionales. Concretamente está compuesto por 10 subescalas que son: Actitud, motivación, control del tiempo, ansiedad, concentración, procesamiento de la información, ayudas para el estudio, autovaloración y estrategias de aprendizaje.

- Escalas de Estrategias de Aprendizaje (ACRA) (Román y Gallego, 1994), dirigidas a evaluar la utilización de estrategias cognitivas por parte de estudiantes de educación secundaria. Está compuesta por cuatro escalas (adquisición, codificación, recuperación y apoyo).

- Cuestionario de las estrategias motivacionales de Self-handicapping (ponerse trabas), Autoafirmación y Pesimismo Defensivo (Suárez, 2000).

- Diagnóstico Integral del Estudio (DIE) (Avellaneda y col., 2000), el cual evalúa hábitos, técnicas y estrategias de estudio en estudiantes desde los 9 años de edad hasta la etapa universitaria. Incluye también una prueba práctica para evaluar la capacidad de estudio real.

- Cuestionario de Hábitos y Técnicas de Estudio (CHTE) (Álvarez y Fernández, 1990) el cual incluye la evaluación de hábitos y técnicas referidas tanto a condiciones físicas y ambientales, como a planificación, gestión del tiempo y utilización de técnicas básicas de estudio.

- Escalas de orientación de meta de Skaalvik (1997) dirigidas a evaluar las orientaciones a meta de los estudiantes, diferenciando las vertientes de orientación a la tarea, de autoensalzamiento del ego, autofrustración del ego y de evitación del esfuerzo.

\section{Conclusiones}

Para finalizar, todo lo tratado hasta aquí tiene una serie de implicaciones tanto para la figura del profesor como para la del orientador, especialmente en los niveles de secundaria y universidad. Muchas de estas implicaciones ya han sido indicadas o bien se pueden extraer 
fácilmente a lo largo de la lectura de este trabajo. Es por ello, junto a las lógicas limitaciones en la extensión del trabajo, que señalaremos únicamente algunas de las implicaciones más generales que se pueden destacar:

- El papel relevante que el AAR adjudica a la consideración de los procesos de pensamiento de los alumnos, incluyendo tanto los elementos más puramente cognitivos como los de tipo afectivo-motivacional.

- La consideración del alumno como protagonista de su proceso de aprendizaje, y por tanto el papel activo del estudiante, en especial el dirigido a la autorregulación y al establecimiento y consecución de metas en las dos vertientes señaladas en el punto anterior.

- Todo lo anterior no implica obviar la importancia del profesor y su papel facilitador en dicho proceso. Muy al contrario, el docente ha de ser conocedor de los diversos tipos de procesos, tanto cognitivos y afectivo-motivacionales que el alumno pone en juego como también de la gestión que éstos realizan sobre sus entornos de aprendizaje. Para ello, se considera útil el planteamiento presentado en este trabajo, el cual proporciona una visión de referencia global desde el que partir en la actividad orientadora sobre el aprendizaje.

- Pero, además del bagaje teórico necesario, los profesionales han de poder contar con una serie de recursos que les permitan realizar una adecuada evaluación y diagnóstico sobre la situación de sus alumnos/as a tal respecto. El conocimiento de las características propias de cada metodología y de cada instrumento, no sólo en cuanto al tipo de variables que aborda, sino también en relación con su fiabilidad y validez, determinarán lo adecuados que pueden resultar para el caso que nos ocupe.

- El fin último de todo ello ha de ser el incidir tanto directa como indirectamente, en el segundo caso por medio del fomento de la propia autorregulación ejercida por parte del estudiante, en el desarrollo de lo que también se ha denominado como aprender a aprender, término que queda adecuadamente redefinido y ampliado bajo el concepto de AAR.

\section{Referencias bibliográficas}

Álvarez, M. y Fernández, R. (1990). Cuestionario de Hábitos y Técnicas de Estudio. Madrid: TEA. Anaya, D. (2002). Diagnóstico en Educación. Madrid: Sanz y Torres.

Avellaneda, M. P., Corps, E. R., Cabezas, M. N. y Polo, A. (2000). Diagnóstico Integral del Estudio. Madrid: TEA.

Beltrán, J. (1993). Procesos, estrategias y técnicas de aprendizaje. Madrid: Síntesis Psicología.

Boekaerts, M. (1996). Self-regulated learning at the junction of cognition and motivation. European Psychologist, 2, 100-112.

Boekaerts, M. (2001). Bringing about change in the classroom: strengths and weaknesses of the selfregulated learning approach. Learning and Instruction, 12 (6), 589-604.

Borkowski, J. G. y Muthukrishna, N. (1992). Moving metacognition into the classroom: "Working models" and effective strategy teaching. En M. Pressley, K. R. Harris y J. T. Guthrie (Eds.), Promoting academic competence and literacy in school. San Diego: Academic Press. 
Bouffard, T., Boisvert, J., Vezeau, C. y Laurache, C. (1995). The impact of goal orientation on self-regulation and performance among college students. British Journal of Educational Psychology, 65, 317-329.

Corno, L. (1986). The metacognitive control components of Self-regulated Learning. Contemporary Educational Psychology, 11, 333-346.

Corno, L. (1995). Comments on Winne: Analytic and systemic research are both needed. Educational Psychologist, 30 (4), 201-206.

Entwistle, N. (1995). Frameworks for understanding as experienced in essay writing and in preparing for examinations. Educational Psycholgist, 30, 47-54.

Garcia, T. y Pintrich, P. R. (1994). Regulating motivation and cognition in the classroom: The role of self-schemas and self-regulatory strategies. En D. H. Schunk y B. J. Zimmerman (Eds.), Self-regulation of learning and performance: Issues and educational applications. Hillsdale, New Jersey: Lawrence Erlbaum Associates.

Pintrich, P. R. (Ed.) (1995). Understanding self-regulated learning. San Francisco: Jossey-Bass Publishers.

Pintrich, P. R. y De Groot, E. V. (1990). Motivational and self-regulated learning components of classroom academic performance. Journal of Educational Psychology, 82 (1), 33-40.

Pintrich, P. R. y García, T. (1991). Student goal orientation and self-regulation in the college classroom. En M. Maehr y P. R. Pintrich (Eds.), Advances in motivation and achievement: Vol. 7. Goals and self-regulatory processes. Greenwich, CT: TAI.

Pintrich, P. R., Roeser, R. W. y De Groot, E. V. (1994). Classroom and individual differences in early adolescents' motivation and self-regulated learning. Journal of Early Adolescence, 14 (2), 139-161.

Pintrich, P. R., Smith, D. A., Garcia, T. y Mckeachie, W. J. (1991). A manual for the use of the Motivated Strategies for Learning Questionnaire (M.S.L.Q.). Ann Arbor, MI: NCRIPTAL: The University of Michigan.

Pokay, P. y Blumenfeld, P. C. (1990). Predicting achievement early and late en the semester: the role of motivation and use of learning strategies. Journal of Educational Psychology, 82 (1), 41-50.

Roces, C., Tourón, J. y González, M. C. (1995). Validación Preliminar del CEAM II (Cuestionario de Estrategias de Aprendizaje y Motivación II). Psicológica, 16 (3), 347-366.

Román, J. M. y Gallego, S. (1994). Estrategias de Aprendizaje. Madrid: TEA.

Schmek, R. R.; Geisler-Brenstein, E. y Cercy, S. P. (1991). Self-concept and learning: the revised inventory of learning processes. Educational Psychology, 11, 343-362.

Schunk, D. H. (1994). Self-regulation of self-efficacy and attributions in academic settings. En D. H. Schunk y B. J. Zimmerman (Eds.), Self-regulation of learning and performance: Issues and educational applications. Hillsdale, New Jersey: Lawrence Erlbaum Associates.

Sheldon, K. M. y Elliot, A. J. (1998). Not all personal goals are personal: Comparing autonomous and controlled reasons for goals as predictors of effort and attainment. Personality and Social Psychology Bulletin, 24 (5), 546-557.

Skaalvik, E. M. (1997). Self-enhancing and self-defeating ego orientation: Relations with task and avoidance orientation, achievement, self-perceptions, and anxiety. Journal of Educational Psychology, 89 (1), 71-81.

Suárez, J. M. (2000). Características diferenciales y predictivas del Aprendizaje Autorregulado en estudiantes universitarios. Tesis doctoral no publicada. Facultad de Ciencias de la Educación. Universidad de A Coruña. 
Suárez, J. M., González, R., Abalde, E. y Valle, A. (2001). Un modelo explicativo de las influencias de las orientaciones de meta sobre la autorregulación del aprendizaje. Revista de Investigación Educativa, 19 (1), 249-262.

Suárez, J. M., González, R. y Valle, A. (2001). Multiple-goal pursuit and its relation to cognitive, selfregulatory, and motivational strategies. British Journal of Educational Psychology, 71, 561-572.

Vermunt, J. D. y Verloop, N. (1999). Congruence and friction between learning and teaching. Learning and Instruction, 9, 257-280.

Weinstein, C. E. (1987). LASSI (Learning and Study Strategies Inventory). Clearwater, FL.: H. \& H. Publishing Co.

Weinstein, C. E. y Mayer, R. E. (1986). Teaching of learning strategies. En M. C. Wittrock (Ed.), Handbook of research on teaching. N. York: Macmillan.

Winne, P. H. (1995). Inherent details in self-regulated learning. Educational Psychologist, 30(4), 173-187.

Wolters, C. A. (1998). Self-regulated learning and college students' regulation of motivation. Journal of Educational Psychology, 90 (2), 224-235.

Zimmerman, B. J. (1994). Dimensions of academic self-regulation: A conceptual framework for education. En D. H. Schunk y B. J. Zimmerman (Eds.), Self-regulation of learning and performance: Issues and educational applications. Hillsdale, New Jersey: Lawrence Erlbaum Associates.

Zimmerman, B. J. y Martínez-Pons, M. (1986). Development of a structured interview for assessing student use of self-regulated learning strategies. American Educational Research Journal, 23, 614-628.

Zimmerman, B. J. y Martinez-Pons, M. (1988). Construct validation of a strategy model of student self-regulated learning. Journal of Educational Psycholgy, 80, 284-290.

Zimmerman, B. J. y Schunk, D. H. (1989). Self-regulated Learning and academic achievement. Theory, research and practice. New York: Springer-Verlag.

Fecha de recepción: 13-10-04

Fecha de revisión: 15-12-05

Fecha de aceptación: 25-04-06 